\title{
Synchronous and Asynchronous Theta and Gamma Activity during Episodic Memory Formation
}

\author{
John F. Burke, ${ }^{1 \star}$ Kareem A. Zaghloul, ${ }^{3 \star}$ Joshua Jacobs, ${ }^{4}$ Ryan B. Williams, ${ }^{2}$ Michael R. Sperling, ${ }^{5}$ Ashwini D. Sharan, ${ }^{6}$ \\ and Michael J. Kahana ${ }^{2}$ \\ ${ }^{1}$ Neuroscience Graduate Group and 2Department of Psychology, University of Pennsylvania, Philadelphia, Pennsylvania 19104, ${ }^{3}$ Surgical Neurology Branch, \\ National Institute of Neurological Disorders and Stroke, National Institutes of Health, Bethesda, Maryland 20892, ${ }^{4}$ School of Biomedical Engineering, \\ Science and Health Systems, Drexel University, Philadelphia, Pennsylvania 19104, and Departments of ${ }^{5}$ Neurology and ${ }^{6}$ Neurological Surgery, Thomas \\ Jefferson University Hospitals, Philadelphia, Pennsylvania 19107
}

To test the hypothesis that neural oscillations synchronize to mediate memory encoding, we analyzed electrocorticographic recordings taken as 68 human neurosurgical patients studied and subsequently recalled lists of common words. To the extent that changes in spectral power reflect synchronous oscillations, we would expect those power changes to be accompanied by increases in phase synchrony between the region of interest and neighboring brain areas. Contrary to the hypothesized role of synchronous gamma oscillations in memory formation, we found that many key regions that showed power increases during successful memory encoding also exhibited decreases in global synchrony. Similarly, cortical theta activity that decreases during memory encoding exhibits both increased and decreased global synchrony depending on region and stage of encoding. We suggest that network synchrony analyses, as used here, can help to distinguish between two major types of spectral modulations: (1) those that reflect synchronous engagement of regional neurons with neighboring brain areas, and (2) those that reflect either asynchronous modulations of neural activity or local synchrony accompanied by global disengagement from neighboring regions. We show that these two kinds of spectral modulations have distinct spatiotemporal profiles during memory encoding.

\section{Introduction}

Studies examining the electrophysiological correlates of memory encoding have demonstrated power fluctuations in theta (3-8 $\mathrm{Hz})$ and gamma $(>40 \mathrm{~Hz})$ frequency bands that reliably covary with episodic memory formation (Kahana, 2006; Nyhus and Curran, 2010). One interpretation of these changes in spectral power is that they reflect oscillatory activity that mediates memory formation through phase synchronization (Axmacher et al., 2006; Jensen et al., 2007; Jutras and Buffalo, 2010; Fell and Axmacher, 2011).

Despite experimental support in humans (Weiss and Rappelsberger, 2000; Fell et al., 2001; Summerfield and Mangels, 2005),

Received April 29, 2012; revised 0ct. 26, 2012; accepted Nov. 2, 2012.

Author contributions: J.F.B., K.A.Z., J.J., and M.J.K. designed research; J.F.B. and R.B.W. performed research; M.R.S., A.D.S., and M.J.K. contributed unpublished reagents/analytic tools; J.F.B. and K.A.Z. analyzed data; J.F.B., K.A.Z., J.J., R.B.W., M.R.S., A.D.S., and M.J.K. wrote the paper.

This work was supported by National Institutes of Health Grants MH055687, MH061975, NS067316, and 5T32MH017168-28 and the Dana Foundation and in part by an Alpha Omega Alpha Carolyn L. Kuckein Student Research Fellowship (J.F.B.). We thank Dale H. Wyeth and Edmund Wyeth for technical assistance at Thomas Jefferson University Hospital and Lynn Lohnas, Ashwin Ramayya, and Nicole Long for helpful discussion and input. We are indebted to all patients who have selflessly volunteered their time to participate in our study.

*JFB. and K.A.Z. contributed equally to this work.

The authors declare no competing financial interests.

Correspondence should be addressed to either of the following: Dr. Michael J. Kahana, Department of Psychology, University of Pennsylvania, 3401 Walnut Street, Room 303C, Philadelphia, PA 19104. E-mail: kahana@psych.upenn.edu; or Dr. Kareem A. Zaghloul, Surgical Neurology Branch, National Institute of Neurological Disorders and Stroke, National Institutes of Health, 10 Center Drive, Room 3D20, Bethesda, MD 20892, E-mail: kareem.zaghloul@nih.gov.

DOI:10.1523/JNEUROSCI.2057-12.2013

Copyright $\odot 2013$ the authors $\quad 0270-6474 / 13 / 330292-13 \$ 15.00 / 0$ the interpretation that episodic memory formation is mediated through phase synchronization is complicated by two observations. First, although localized increases in theta activity have been demonstrated in the temporal cortex and hippocampus (Klimesch et al., 1996; Mölle et al., 2002; Sederberg et al., 2003; Hanslmayr et al., 2011; Lega et al., 2011), memory encoding is also associated with an extensive decrease in theta power (Sederberg et al., 2007a; Guderian et al., 2009). Second, the observed increases in gamma power during successful memory encoding occur across a wide range of frequencies extending from 30 to $100 \mathrm{~Hz}$ (Sederberg et al., 2007b). Such broadband activity is inconsistent with a mechanism that relies on precise narrowband phase synchronization (Ray and Maunsell, 2010). An alternative hypothesis that has gained traction outside of the memory literature is that the observed increases in gamma activity often reflect an arrhythmic and intrinsically asynchronous process (Manning et al., 2009; Miller et al., 2009; Ray and Maunsell, 2011).

To disentangle whether the observed changes in spectral power reflect oscillatory or asynchronous activity, it is necessary to directly measure phase synchrony during successful versus unsuccessful memory encoding. In particular, if oscillations serve to bind a spatially distributed memory representation, overall synchrony should increase. Conversely, if asynchronous activity dominates the changes in spectral power, synchrony will likely decrease. Previous studies examining theta and gamma functional connectivity during memory formation have limited the search for phase synchrony to specific anatomical circuits hy- 
pothesized to participate in memory encoding (Fell et al., 2001). Power fluctuations during memory formation, however, appear across widespread cortical and subcortical regions. Determining whether these spatially distributed power fluctuations represent synchronous oscillatory activity requires the implementation of analysis techniques specifically designed to aggregate large-scale network connections (Varela et al., 2001; Bullmore and Sporns, 2009; Siegel et al., 2012).

Here, we use recordings from intracranial electrodes implanted in 68 participants engaging in a free-recall task to simultaneously investigate changes in spectral power and phase during successful and unsuccessful memory encoding. We use a regionof-interest (ROI) approach and a graph-theoretic metric of phase synchrony to localize both oscillatory and asynchronous activity to interpret the observed changes in spectral power. We find specific time intervals and anatomical locations that exhibit changes in both theta and gamma synchrony during memory formation. By separating phase-synchronous oscillations from asynchronous changes, our results help clarify the role of rhythmic neural activity in the memory system.

\section{Materials and Methods}

Participants. Seventy-six participants with medication-resistant epilepsy underwent a surgical procedure in which platinum recording contacts were implanted subdurally on the cortical surface, as well as deep within the brain parenchyma. In each case, the clinical team determined the placement of the contacts so as to best localize epileptogenic regions. Data were collected at four different hospitals: Boston Children's Hospital (Boston, MA), Hospital of the University of Pennsylvania (Philadelphia, PA), Freiburg University Hospital (Freiburg, Germany), and Thomas Jefferson University Hospitals (Philadelphia, PA). Our research protocol was approved by the institutional review board at each hospital, and informed consent was obtained from the participants and their guardians. We restricted our analysis to include only those patients who were left-hemispheric language dominant, as assessed by the patients' handedness, a clinically administered intracarotid injection of sodium amobarbital (Wada test), or functional magnetic resonance imaging (MRI) using a verb-generation task (Thomas Jefferson University Hospitals). Our final participant pool consisted of 68 patients ( 27 females; Table 1).

Free-recall task. Each patient participated in a delayed free-recall task (Fig. 1A). In each trial of this task, participants are instructed to study a list of words and are then asked to freely recall as many words as possible. Lists were composed of either 15 (54 of 68 patients) or 20 common nouns, chosen at random and without replacement from a pool of highfrequency nouns (http://memory.psych.upenn.edu/WordPools). Words were presented sequentially and appeared in capital letters at the center of the screen. Each word remained on the screen for $1600 \mathrm{~ms}$, followed by a randomly jittered $800-1200 \mathrm{~ms}$ blank interstimulus interval (ISI). The random duration of the ISI served to decorrelate the physiological responses from successive word presentations.

After the final word in each list, participants were given a distraction task designed to attenuate any advantage in recalling the most recently studied items (Kahana, 2012). The distraction task was a series of arithmetic problems of the form $A+B+C=$ ??, where $A, B$, and $C$ were randomly chosen integers ranging from 1 to 9 . The distraction interval lasted at least $20 \mathrm{~s}$, but patients were allowed to complete any problem that they started, resulting in a variable distraction interval (average duration was $22.7 \mathrm{~s}$ ).

After the distraction period, participants were given $45 \mathrm{~s}$ to recall as many words as possible from the list in any order. Vocalizations were digitally recorded and subsequently manually scored for analysis. Words that were presented during the encoding period and successfully retrieved during the recall period were considered successfully encoded (e.g., the words DOG, TREE, and LIME in Fig. 1A). Likewise, words that were not retrieved during the recall period were considered unsuccessfully encoded (e.g., the words CAT and BALL in Fig. 1A).
Electrocorticographic recordings. Data from our 68-patient database were collected over a 13-year period in collaboration with four different hospitals. Whereas each hospital used the same general implantation procedures and data-acquisition techniques, our analysis had to account for technical details that varied by institution. Electrocorticography (ECoG) data were recorded using a Bio-Logic, DeltaMed, Nicolet, GrassTelefactor, or Nihon Kohden electroencephalogram (EEG) system. Depending on the amplifier and the discretion of the clinical team, the signals were sampled at $256,400,500,512,1000,1024$, or $2000 \mathrm{~Hz}$. Signals were referenced to a common contact placed either intracranially or on the scalp or mastoid process. All recorded traces were resampled at $256 \mathrm{~Hz}$, and a fourth-order 2-Hz stop-band Butterworth notch filter was applied at either 60 or $50 \mathrm{~Hz}$ to eliminate electrical line noise. The testing laptop sent $\pm 5 \mathrm{~V}$ analog pulses, via an optical isolator, into a pair of open lines on the clinical recording system to synchronize the electrophysiological recordings with behavioral events.

We collected electrophysiological data from surgically implanted subdural and depth recording intracranial contacts (Fig. 1B). Subdural contacts were arranged in both grid and strip configurations with an inter-contact spacing of $10 \mathrm{~mm}$. Depth contacts (six to eight linearly arranged contacts spaced $8 \mathrm{~mm}$ apart) were placed in 46 of 68 patients; all depth contacts were placed in the medial temporal lobe, except for one patient whose depths were placed in the superior temporal gyrus near auditory cortex (TJUH 17; Table 1). Contact localization was accomplished by coregistering the postoperative computed tomographies (CTs) with the postoperative MRIs using FSL [FMRIB (Functional MRI of the Brain) Software Library] BET (Brain Extraction Tool) and FLIRT (FMRIB Linear Image Registration Tool) software packages. Preoperative MRIs were used when postoperative MRIs were not available. The resulting contact locations were mapped to both Montreal Neurological Institute (MNI) and Talairach space using an indirect stereotactic technique and OsiriX Imaging Software DICOM viewer package (Fig. 1C). Details regarding each patient's montage, behavioral performance, and amplifier filter settings can be found in Table 1 .

Two concerns when analyzing bivariate interactions between closely spaced intracranial contacts are volume conduction and confounding interactions with the reference line. We used bipolar referencing to eliminate such confounds when analyzing the neural signal (Nunez and Srinivasan, 2006). We defined the bipolar montage in our dataset based on the geometry of ECoG electrode arrangements. For every grid, strip, and depth probe, we isolated all pairs of contacts that were positioned immediately adjacent to one another; bipolar signals were then found by differencing the signals between each pair of immediately adjacent contacts (Anderson et al., 2010). The resulting bipolar signals were treated as new virtual electrodes (henceforth referred to as electrodes throughout the text), originating from the midpoint between each contact pair (Fig. 1C, red circles). All subsequent analyses were performed using these derived bipolar signals. We excluded pairs sharing a common contact when calculating synchrony to remove all confounding interactions attributable to shared information. In total, our dataset consisted of 6946 electrodes (3237 left hemispheric; 3709 right hemispheric).

Data analyses and spectral power. To quantify memory-related changes in spectral power and synchrony, we convolved the downsampled (256 $\mathrm{Hz}$ ) bipolar ECoG signals with complex-valued Morlet wavelets (wave number 10) to obtain magnitude and phase information (Addison, 2002). We used six wavelets with center frequencies spaced $1 \mathrm{~Hz}$ apart within the theta $(3-8 \mathrm{~Hz})$ frequency range and 11 wavelets with center frequencies spaced $5 \mathrm{~Hz}$ apart within the gamma $(45-95 \mathrm{~Hz})$ frequency range. Each wavelet was convolved with $3750 \mathrm{~ms}$ of ECoG data surrounding each word presentation, from $1000 \mathrm{~ms}$ before word onset to $2750 \mathrm{~ms}$ after word onset (a $1000 \mathrm{~ms}$ buffer was included on both sides of the clipped data). We subsequently binned the continuous-time transforms into $1000 \mathrm{~ms}$ epochs with $75 \%$ overlap, yielding 12 total temporal epochs surrounding each word presentation. One-thousand-millisecond epochs were chosen so that each window contained at least three cycles of the lowest frequency analyzed $(3 \mathrm{~Hz})$.

To assess memory-related changes in spectral power within theta and gamma frequencies, we squared and log-transformed the magnitude of the continuous-time wavelet transform to generate a continuous mea- 
Table 1. EEG free-recall patient database

\begin{tabular}{|c|c|c|c|c|c|c|}
\hline$\overline{\mathrm{ID}}$ & Gender & Age (years) & $\% \operatorname{Rec}$ & \#BPD & Pass band (Hz) & Electrode coverage \\
\hline CHB 1 & M & 15 & 28.7 & 200 & $0.1-100$ & L frontal grids + strips \\
\hline CHB 2 & $\mathrm{~F}$ & 8 & 26.5 & 99 & $0.1-100$ & R temporal grids; L temporofrontal strips \\
\hline CHB 3 & M & 17 & 10.7 & 105 & $0.1-100$ & L temporal grids + strips \\
\hline CHB 4 & $\mathrm{~F}$ & 20 & 31.7 & 184 & $0.1-100$ & $\mathrm{~b} / \mathrm{l}$ frontal grids + strips \\
\hline CHB 5 & M & 14 & 20.9 & 128 & $0.1-100$ & R parieto- occipital grids \\
\hline CHB 6 & $\mathrm{~F}$ & 19 & 18.4 & 172 & $0.1-100$ & R frontoparietal grids \\
\hline CHB 7 & M & 16 & 31.7 & 232 & $0.1-100$ & L frontal grids + strips \\
\hline CHB 8 & M & 13 & 33.3 & 103 & $0.1-100$ & L frontotemporal grids \\
\hline FRUH 1 & M & 33 & 23.9 & 108 & $0.032-97$ & R grid; R depth; b/l strips \\
\hline FRUH 2 & M & 25 & 25.0 & 131 & $0.032-97$ & $R$ frontoparietal grid \\
\hline FRUH 3 & M & 31 & 13.3 & 72 & $0.032-97$ & L depth; L temporal grid + strips \\
\hline FRUH 4 & $\mathrm{~F}$ & 41 & 16.4 & 110 & $0.032-97$ & R frontoparietal grid \\
\hline FRUH 5 & $\mathrm{~F}$ & 34 & 27.1 & 42 & $0.032-97$ & $b / l$ depth + strips \\
\hline FRUH 6 & $\mathrm{~F}$ & 45 & 16.9 & 152 & $0.032-97$ & L frontoparietal grid \\
\hline FRUH 7 & $\mathrm{~F}$ & 46 & 16.7 & 20 & $0.032-97$ & L depths \\
\hline FRUH 8 & M & 20 & 14.0 & 124 & $0.032-97$ & R parieto-occipito-temporal grid \\
\hline FRUH 9 & $\mathrm{~F}$ & 53 & 16.3 & 44 & $0.032-97$ & b/l depths; $L$ temporal strips \\
\hline FRUH 10 & M & 50 & 19.3 & 90 & $0.032-97$ & L depth; L temporal grid \\
\hline FRUH 11 & M & 28 & 12.3 & 171 & $0.032-97$ & L fronto-temporo-parietal grids \\
\hline FRUH 12 & $\mathrm{~F}$ & 37 & 21.7 & 39 & $0.032-97$ & $\mathrm{~b} / \mathrm{l}$ depths \\
\hline FRUH 13 & M & 18 & 40.3 & 34 & $0.032-97$ & $\mathrm{~b} / \mathrm{l}$ depths \\
\hline FRUH 14 & M & 23 & 34.5 & 77 & $0.032-97$ & L depth; L temporal grid + strips \\
\hline FRUH 15 & M & 21 & 32.7 & 144 & DC-344 & R temporo-occipito-parietal grid + strips \\
\hline FRUH 16 & $\mathrm{~F}$ & 35 & 18.0 & 181 & $0.032-97$ & $R$ depth; $R$ fronto-temporo-parietal grid \\
\hline FRUH 17 & $\mathrm{~F}$ & 37 & 30.7 & 69 & DC-344 & $R$ depth; $R$ fronto-temporo-parietal grid \\
\hline FRUH 18 & $\mathrm{~F}$ & 41 & 8.4 & 44 & $0.032-97$ & b/l depths \\
\hline FRUH 19 & $\mathrm{~F}$ & 21 & 22.2 & 112 & DC-344 & R frontal grid \\
\hline FRUH 20 & $\mathrm{~F}$ & 43 & 10.2 & 65 & DC-344 & $\mathrm{b} / \mathrm{l}$ depths + temporal strips \\
\hline FRUH 21 & M & 19 & 32.0 & 44 & $0.032-97$ & $\mathrm{~b} / \mathrm{l}$ depths \\
\hline FRUH 22 & M & 21 & 51.7 & 59 & DC-344 & R depths; $R$ temporofrontal strips \\
\hline FRUH 23 & $\mathrm{~F}$ & 35 & 19.6 & 87 & $0.032-97$ & L temporal grid, strips, depths \\
\hline FRUH 24 & M & 25 & 32.3 & 134 & $0.032-97$ & L frontal grid \\
\hline FRUH 25 & $\mathrm{~F}$ & 41 & 28.7 & 92 & $0.032-97$ & b/l depths; occipitofrontal strips \\
\hline TJUH 1 & M & 25 & 32.2 & 60 & $0.5-100$ & L depths; L temporofrontal strips \\
\hline TJUH 2 & $\mathrm{~F}$ & 40 & 17.1 & 88 & $0.5-100$ & b/l depths; b/l temporal strips \\
\hline TJUH 3 & $\mathrm{~F}$ & 34 & 22.7 & $95^{a}$ & $0.5-100$ & $\mathrm{~b} / \mathrm{l}$ depths; $\mathrm{R}$ temporo-fronto-parietal strips \\
\hline TJUH 4 & M & 44 & 15.9 & 110 & $0.5-100$ & $\mathrm{~b} / \mathrm{l}$ depths; $\mathrm{b} / \mathrm{l}$ temporofrontal strips \\
\hline TJUH 5 & M & 43 & 24.2 & 68 & $0.5-100$ & b/I depths; R temporo-occipital strips \\
\hline TJUH 6 & M & 21 & 20.1 & 129 & $0.5-100$ & b/l frontotemporal grid + strips; L depths \\
\hline TJUH 7 & M & 56 & 16.7 & 43 & $0.5-100$ & L depths; L temporo-occipital strips \\
\hline TJUH 8 & $\mathrm{~F}$ & 57 & 10.0 & 81 & $0.03-600$ & L depths; L temporal grid + strips \\
\hline TJUH 9 & M & 20 & 26.7 & $139^{a}$ & $0.03-300$ & b/l depths; b/l temporofrontal strips \\
\hline TJUH 10 & M & 41 & 20.8 & 84 & $0.03-600$ & R depths; $R$ temporo-parieto-occipital strips \\
\hline TJUH 11 & $\mathrm{~F}$ & 34 & 26.7 & 95 & $0.03-600$ & L depths + temporal grid + strips \\
\hline TJUH 12 & $\mathrm{~F}$ & 52 & 36.7 & 95 & $0.03-600$ & Rdepths + temporofrontal grid \\
\hline TJUH 13 & M & 44 & 45.0 & 92 & $0.03-600$ & $R$ depths; $b /$ l temporofrontal strips \\
\hline TJUH 14 & M & 33 & 32.0 & 105 & $0.03-600$ & b/l temporo-fronto-parietal strips \\
\hline TJUH 15 & $\mathrm{~F}$ & 23 & 29.2 & 94 & $0.03-600$ & R depths + grid; b/l temporal strips \\
\hline TJUH 16 & $\mathrm{~F}$ & 48 & 35.0 & 84 & $0.03-600$ & $\mathrm{~b} / \mathrm{I}$ temporo-fronto-occipital strips \\
\hline TJUH 17 & M & 33 & 37.6 & 46 & $0.03-1200$ & L temporal strips + superior temporal gyrus depths \\
\hline TJUH 18 & M & 45 & 22.6 & 86 & $0.03-600$ & b/l depths; $b$ /l temporofrontal strips \\
\hline TJUH 19 & M & 23 & 37.2 & 78 & $0.03-600$ & R depths; $R$ temporo-fronto-parietal strips \\
\hline TJUH 20 & M & 53 & 18.1 & 83 & $0.03-600$ & b/l depths; b/l temporal strips \\
\hline TJUH 21 & M & 29 & 48.3 & 63 & $0.03-600$ & $\mathrm{~b} / \mathrm{l}$ temporal $+\mathrm{L}$ fronto-occipital strips \\
\hline TJUH 22 & M & 35 & 22.8 & 90 & $0.03-600$ & b/l frontotemporal strips; $b / l$ depths \\
\hline TJUH 23 & $\mathrm{~F}$ & 48 & 18.0 & $130^{a}$ & $0.03-600$ & b/l strips (all lobes); b/l depths \\
\hline TJUH 24 & $\mathrm{~F}$ & 20 & 43.1 & 126 & $0.03-600$ & $\mathrm{~b} / \mathrm{I}$ strips (all lobes); $R$ depths \\
\hline TJUH 25 & M & 20 & 30.8 & $63^{a}$ & $0.03-600$ & R temporo-occipital strips; $R$ depths \\
\hline HUP 1 & M & 38 & 22.5 & 69 & $0.16-134$ & R depths; $R$ temporo-occipito-frontal strips \\
\hline HUP 2 & M & 30 & 18.0 & 72 & $0.16-134$ & b/l depths; b/l frontotemporal strips \\
\hline HUP 3 & M & 43 & 11.5 & 97 & $0.16-134$ & R frontal grid; R temporal strips \\
\hline HUP 4 & M & 36 & 11.7 & 75 & $0.16-134$ & b/l depths; b/l temporofrontal strips \\
\hline HUP 5 & M & 25 & 22.5 & 53 & $0.16-134$ & R depths; $R$ strips \\
\hline HUP 6 & $\mathrm{~F}$ & 18 & 23.1 & 64 & $0.16-134$ & b/l depths; b/l frontotemporal strips \\
\hline HUP 7 & $\mathrm{~F}$ & 27 & 21.7 & 40 & $0.16-134$ & b/l depths; b/l frontotemporal strips \\
\hline HUP 8 & $M$ & 40 & 31.7 & 47 & $1.6-134$ & b/l temporo-fronto-parietal strips \\
\hline HUP 9 & M & 27 & 28.1 & 68 & $1.6-134$ & b/l depths; b/l frontotemporal strips \\
\hline HUP 10 & M & 37 & 25.1 & 123 & $1.6-134$ & R temporo-fronto-parietal grid + strips \\
\hline
\end{tabular}

For each participant, the identification number (ID), gender, age, percentage of correctly encoded words (\% Rec), number of bipolar electrode pairs (\#BPD), pass-band of the filter settings of the amplifier, and a brief anatomical description of the electrode coverage are listed. CHB, Boston Children's Hospital; FRUH, Freiburg University Hospital; TJUH, Thomas Jefferson University Hospitals; HUP, Hospital of the University of Pennsylvania; F, female; M, male; DC, amplifiers allowed direct current signal to be recorded; $L$, left; $R$, right; $b / l$, bilateral.

${ }^{a}$ The electrode montage was changed during the hospital stay. The second montages from patients TJUH3, TJUH 9, TJUH 23, and TJUH 25 contained 81, 50, 133, and 140 bipolar derivations, respectively. The third montage from patient TJUH 9 contained 139 bipolar derivations. 

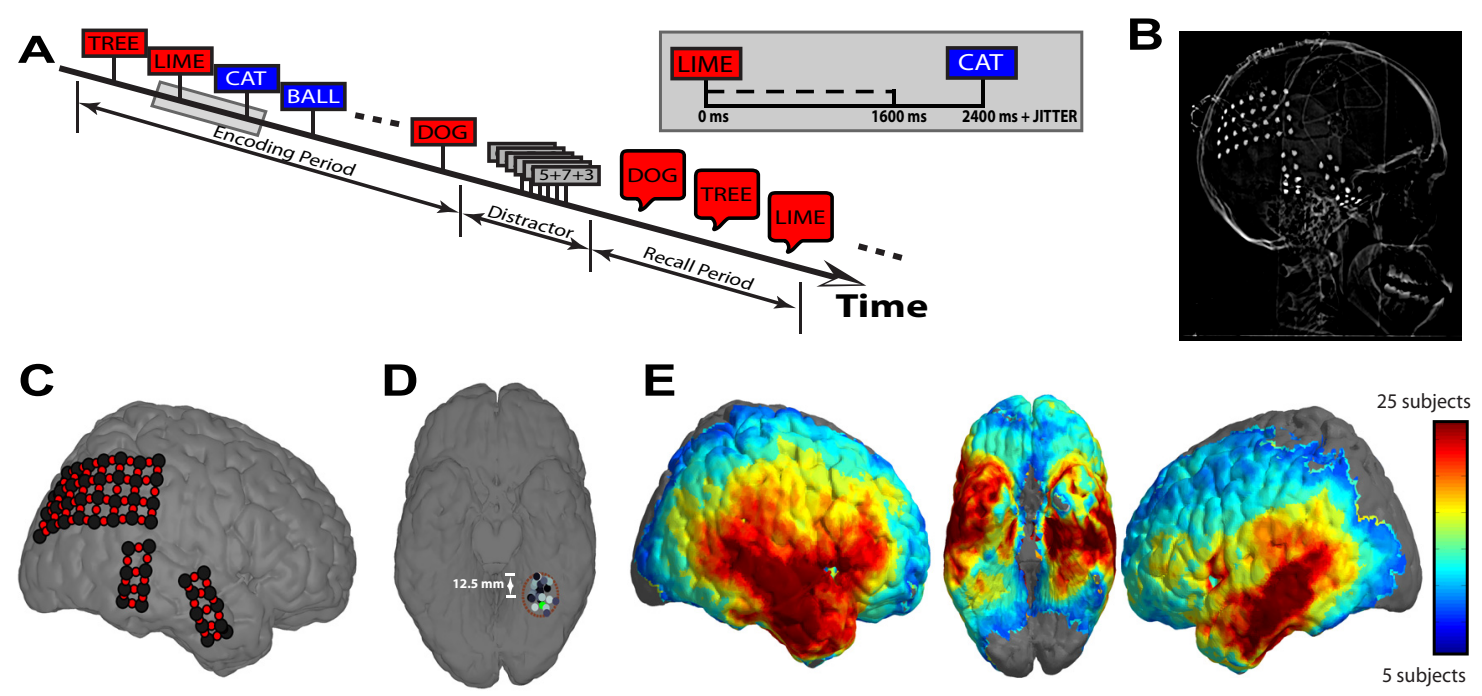

Figure 1. Behavioral task and data collection. A, Participants were shown a list of words during the encoding period and, after a distraction interval, were asked to verbally recall as many words as possible in any order. Words remained on the screen for $1600 \mathrm{~ms}$, after which the screen was left blank for $800 \mathrm{~ms}$ (plus a $0-400 \mathrm{~ms}$ uniformly distributed random temporal jitter) before presentation of the next word. $\boldsymbol{B}$, Example radiographic image of a participant's electrode arrangement. $\boldsymbol{C}$, Electrodes from the participant in $\boldsymbol{B}$ were coregistered to a standardized brain in Talairach space. Bipolar signals (red circles) were found by differencing the voltage traces from immediately adjacent electrodes (black circles). $\boldsymbol{D}$, Colored dots represent electrodes from 15 different participants clustered within a spherical region of $12.5 \mathrm{~mm}$ radius centered at $x=-32.00, y=-52.38, z=-16.25$ in Talairach space (fusiform gyrus). $E$, Heat map showing spatial distribution of electrode locations. The number of patients with at least one electrode within $12.5 \mathrm{~mm}$ of each location on the standardized brain is shown (locations with electrodes from 5 or fewer patients are not colored).

sure of instantaneous power. For each word presentation, we averaged the instantaneous power across each time epoch and did so separately across theta and gamma frequencies. To account for changes in power across sessions, we $z$-transformed power values separately for each session with the mean \pm SD for a set of baseline events, which were $1000 \mathrm{~ms}$ windows spaced every $60 \pm 10 \mathrm{~s}$ during the testing session. For every electrode and temporal epoch, we assessed the difference in spectral power during memory formation by calculating a Welch's parametric $t$ statistic on the distributions of average power values during successful and unsuccessful encoding for both theta and gamma frequencies.

Synchrony and functional connectivity. To obtain an estimate of the synchrony between two electrodes, $e_{p}$ and $e_{q}$, during a single time epoch for a given frequency, $f$, we calculated the phase-locking value $\left(\bar{R}_{p q}\right)$ between their continuous-time phase signals, $\phi_{p}(t, f)$ and $\phi_{q}(t, f)$ (Lachaux et al., 1999):

$$
\bar{R}_{p q}(f)=\frac{1}{N}\left|\sum_{t=1}^{N} e^{i\left(\phi_{p}(t, f)-\phi_{q}(t, f)\right)}\right|,
$$

where $N$ is the total number of samples within the temporal epoch of interest. We averaged $\bar{R}_{p q}(f)$ across all theta and gamma frequencies to generate a theta and gamma phase-locking value, $\bar{R}_{p q}(\theta)$ and $\bar{R}_{p q}(\gamma)$, for each temporal epoch during each word presentation for every electrode pair, $e_{p}, e_{q} \forall p \neq q$ and $p, q \in\{1,2, \ldots P\}$, where $P$ is the total number of electrodes in the montage.

To assess the difference in phase-locking value during memory formation for an individual participant, we used a parametric $t$ test to compare the distribution of phase-locking values during successful and unsuccessful memory encoding for each temporal epoch, frequency band, and electrode pair. Electrode pairs exhibiting statistically significant $(p<$ 0.05 ) increases or decreases in phase-locking value in an individual participant were visualized by rendering red and blue lines, respectively, between each electrode (see Fig. 6A).

To more precisely localize memory-related changes in synchrony, we identified electrodes that either increased or decreased their total connections to all other electrodes during successful encoding using a metric derived from graph theory. We defined every electrode as a node, $e_{p}$, in a network, and every possible connection between that node and every other node, $e_{q}$, as an edge, $K_{p, q}$. Every edge can take on one of three values, depending on whether there was a statistically significant change in the phase-locking value, $\bar{R}$, between any two nodes, $e_{p}$ and $e_{q}$, during successful encoding:

$$
K_{p, q}=\left\{\begin{array}{ll}
+1 & \text { statistically significant increase in } \bar{R} \\
0 & \text { no change in } \bar{R} \\
-1 & \text { statistically significant decrease in } \bar{R}
\end{array} .\right.
$$

For a given node, $e_{p}$, we then define the change in degree, $\Delta d_{p}$, as the net change in the value of all edges connected to that node:

$$
\Delta d_{p}=\sum_{q=1}^{p} K_{p, q} \forall q \neq p,
$$

where $P$ represents the total number of electrodes in the montage. $\Delta d_{p}$ quantifies the extent to which one electrode or node changes its functional connections to all other electrodes during successful encoding. If an electrode, $e_{p}$, participates in many connections with statistically significant increases or decreases in $\bar{R}$, the subsequent change in degree, $\Delta d_{p}$, would be very positive or negative, respectively.

Anatomical localization. To identify whether a particular anatomical area exhibited task-related changes in power or phase synchrony, we grouped spatially similar electrodes from different participants using both an ROI and a voxel-based approach.

In the ROI approach, we segregated electrodes into five anatomical lobes (frontal, temporal, parietal, occipital, and limbic) and one hippocampal region from each hemisphere to generate 12 mutually exclusive ROIs (Lancaster et al., 2000; Manning et al., 2011). For the hippocampal ROI, a clinician experienced in neuroanatomical localization manually reviewed postoperative CTs and MRIs to accurately identify all depth contacts located within the hippocampus (Lega et al., 2011; Serruya et al., 2012). A bipolar pair was categorized into the hippocampal ROI if at least one contact within the pair was determined to lie within this structure, yielding 361 hippocampal electrodes from 44 patients.

In the voxel-based approach, we divided Talairach space into 5484 overlapping 12.5 -mm-radius spheres evenly placed every $6.25 \mathrm{~mm}$ in the $x, y$, and $z$ directions. Only spherical voxels and ROIs with electrodes from five or more patients were included in statistical analyses (Fig. 1E). We averaged statistics within each individual so that a single region was 
not overrepresented by a participant who happened to have many electrodes within that region.

Statistical analyses. We assessed whether changes in spectral power were significant across participants for a given ROI or spherical voxel using a nonparametric permutation procedure. We calculated a $t$ statistic on the distribution of log-power values during successful and unsuccessful encoding for both theta and gamma frequencies during a single temporal epoch for every electrode and from each participant. We then permuted the labels for the conditions $N$ times $(N=2000$ for spherical voxels and $N=20,000$ for ROIs) to generate a distribution of $N$ shuffled $t$ statistics. We averaged the true and permuted $t$ statistics across all electrodes within each ROI and within each spherical voxel for each participant. For each region, we then summed the true and permuted averaged values across all participants (Sederberg et al., 2003, 2007a,b). To generate a $p$ value for changes in spectral power for a given region, we determined the position of the summed true $t$ statistics in the distribution of summed permuted values. Given the relatively small number of regions in the ROI power analysis, multiple comparisons were Bonferroni's corrected across time, frequency band, and ROI.

To assess changes in phase synchrony between ROIs, we used a similar nonparametric permutation procedure. For every ROI pair containing electrode pairs from five or more patients, we calculated the average phase-locking value, $\bar{R}$, across all electrode pairs spanning the two ROIs (or across all electrode pairs within a single ROI) for a given participant. We calculated a $t$ statistic on the distribution of average $\bar{R}$ values during successful and unsuccessful encoding for both theta and gamma frequencies during every temporal epoch. We then permuted the labels for the conditions 20,000 times to generate a null distribution for each $t$ statistic. For each ROI pair, we summed the true and permuted $t$ statistics across all participants and determined the position of the true $t$ statistics in the distribution of summed permuted values. To correct for multiple comparisons across time, frequency, and ROI pairs, a false discovery rate (FDR) procedure was applied using both a conservative $(Q=0.05)$ and more liberal $(Q=0.1)$ threshold (Genovese et al., 2002). ROI pairs exhibiting a statistically significant increase or decrease in phase synchrony were visualized by rendering red and blue lines, respectively (see Fig. 5).

To assess whether more precisely localized changes in phase synchrony were significant across participants, we used a similar nonparametric permutation procedure to examine changes in degree $(\Delta d)$, or functional connectivity, for each spherical voxel. For a given electrode, $e_{p}$, from a single participant, we calculated the change in degree, $\Delta d_{p}$, between successful and unsuccessful encoding for both theta and gamma frequencies during a single temporal epoch. We then permuted the labels for the conditions 2000 times to generate a distribution of 2000 shuffled $\Delta d_{p}$ values. We averaged the true and permuted changes in degree across all electrodes within each $12.5 \mathrm{~mm}$ spherical region for each participant. As we did for power, we then summed the true and permuted averaged changes in degree for each region, across all participants, and determined the position of the summed true values in the distribution of summed permuted values to generate a $p$ value.

Topographic plots. To plot spatial changes in spectral power and synchrony, we identified spherical voxels that exhibited a statistically significant $(p<0.001)$ increase or decrease in power or functional connectivity across participants. At each spherical voxel, we calculated the percentage of spherical voxels with centers within a surrounding region of $12.5 \mathrm{~mm}$ that exhibited identical encoding-related effects. We translated these percentages to color saturation and rendered these values onto cortical and subcortical topographical plots using a standard MNI brain with information from the Wake Forest University PickAtlas toolbox (Maldjian et al., 2003). Increases in power and functional connectivity were rendered with red colors, and decreases were rendered with blue. Colored values were smoothed using a three-dimensional Gaussian kernel (radius $=12.5 \mathrm{~mm} ; \sigma=6.25 \mathrm{~mm}$ ). The maximal color saturation in either direction corresponded to $25 \%$ of local spherical voxels. All regions with fewer than five patients were colored black and were not analyzed. Grayscale rendering in other regions represented the percentage of spherical voxels surrounding a given location with at least five patients and thus represented regions that were analyzed but that did not exhibit significant effects.

\section{Results}

We set out to determine whether power fluctuations that accompany successful memory encoding represent phase-synchronous neural oscillations. We investigated this issue in two data-analytic stages. First, we characterized the anatomical distribution and timing of spectral power changes that correlated with successful encoding. Second, we characterized the degree to which ECoG activity recorded in a given brain region was synchronous with ECoG activity recorded elsewhere in the brain. Of particular interest is whether regions that show increased spectral power in our first analysis show increased or decreased synchrony in our second analysis.

We briefly note that we have operationalized successful encoding by contrasting the ECoG signals measured during encoding of items that are subsequently recalled $(24.9 \%$ of studied items in our delayed free-recall task) with those items that are not subsequently recalled. Whereas this method has been widely used to investigate the neurological basis of memory formation in a number of studies (Paller and Wagner, 2002), we recognize that this operationalization is limited insofar as retrieval factors will account for much of the variation in subsequent recall (Kahana, 2012).

\section{Changes in spectral power during encoding}

We examined changes in theta $(3-8 \mathrm{~Hz})$ and gamma $(45-95 \mathrm{~Hz})$ power after word presentation during successful and unsuccessful memory encoding. We used 5484 identical three-dimensional spherical regions uniformly placed across Talairach space to group spectral activity from nearby electrodes for each participant (Fig. 1D). For each frequency band, we calculated an average $t$ statistic for all electrodes in each region by comparing theta and gamma power between successfully and unsuccessfully remembered items during each temporal epoch after word presentation. To assess whether spectral changes are statistically reliable across participants, we used a permutation procedure to map encodingrelated changes in theta and gamma power to each region (see Materials and Methods). We visualized spherical voxels that exhibited a statistically significant $(p<0.001)$ change in theta and gamma power during successful memory encoding on a standardized three-dimensional brain (Fig. $2 A, B$ ) during two representative temporal epochs: $0-1000 \mathrm{~ms}$ (early word presentation) and 500-1500 ms (late word presentation).

We found a reliable decrease in theta power across several brain regions after the presentation of words that were successfully remembered compared with words that were not remembered (Fig. 2A). The decreases in theta power were more prominent in posterior temporal regions during early word presentation but expanded to include frontal and anterior temporal regions during late word presentation. Whereas decreased theta power with successful encoding was by far the most prevalent pattern observed across cortical and medial temporal regions, smaller increases in theta power were observed in the right anterior temporal lobe and in the left anterior frontal lobe immediately after word presentation.

We found that memory encoding had a very different effect on gamma power compared with theta power (Fig. 2B). Specifically, a large number of regions exhibited a reliable increase in gamma power after the presentation of words that were successfully remembered compared with words that were not remembered. As with theta power, the changes we observed in the gamma band 

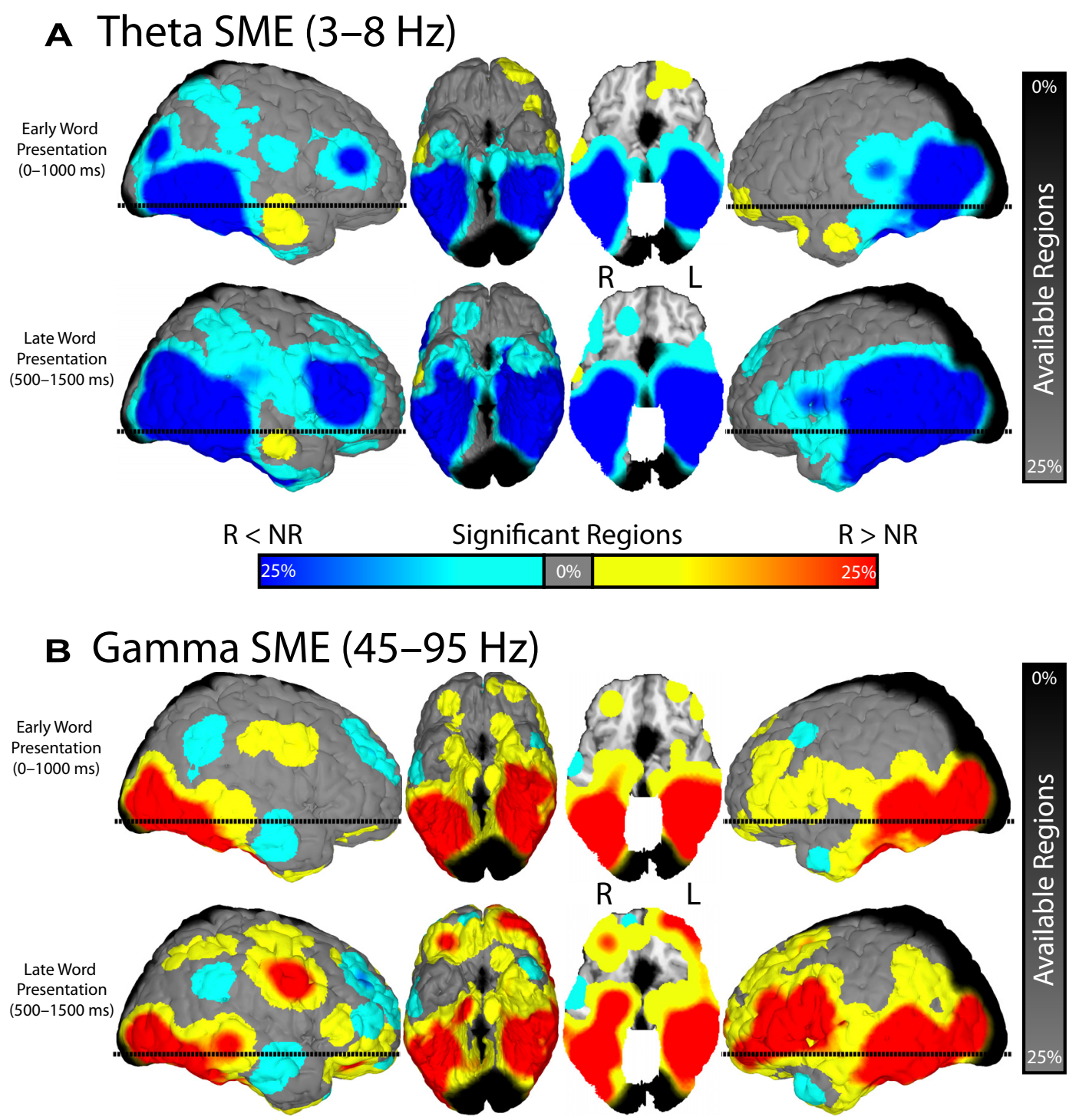

Figure 2. Change in theta and gamma power across anatomical location. For two representative time epochs, $0-1000 \mathrm{~ms}$ (early word presentation) and $500-1500 \mathrm{~ms}$ (late word presentation), all spherical regions that exhibited a significant $(p<0.001)$ change in theta $(\boldsymbol{A})$ and gamma $(\boldsymbol{B})$ power with successful memory encoding (SME) are displayed on a standardized three-dimensional brain. Increases $(R>N)$ and decreases $(N<R)$ in power are shown in red and blue, respectively. $N$, Not recalled; $R$, recalled. The color scale reflects the percentage of nearby ROls exhibiting identical encoding-related effects. The horizontal dashed line on the sagittal views corresponds to the level of the axial cut in the third panel (the $z=-13.0$ plane in Talairach space). Grayscale rendering represents the percentage of spherical voxels surrounding a given location with at least five patients. L, Left; $R$, right.

were more prominent in posterior cortices early after item presentation and expanded anteriorly during later intervals. In addition, the increases we observed in the gamma range lateralized to the left hemisphere; gamma activations in the right hemisphere were more spatially discrete and co-occurred alongside pockets of decreased gamma power. The left-hemispheric bias of gamma activations likely reflects the language comprehension component of our task.

To investigate the temporal evolution of these effects, we separately counted the number of spherical regions that exhibited a statistically significant change in power in each time bin (Fig. $3 A, B)$. Given our significance threshold $(p<0.001)$ and that our analysis was conducted over 5484 spherical regions and two frequency bands, we expected to find 11 total regions showing a significant change in power during any given time epoch by chance (5.5 regions in each tail of the distribution; Fig. $3 A, B$, dashed line). Figure 3 demonstrates that decreases in theta power and increases in gamma power during memory formation far exceeded this expectation and were also tightly linked to item presentation. The precise timing of these effects suggests that the observed changes in power are driven specifically by item presentation as opposed to more nonspecific cognitive processes, such as global shifts in attention.

For each electrode in our database, we also visually examined the raw log-transformed power values during successfully encoded, unsuccessfully encoded, and baseline events. Four representative examples of electrodes that individually show the effects described in Figures 2 and 3 are shown in Figure 4 and highlight two important features in our data. First, the simultaneous increase in gamma power and decrease in theta power that accompany memory formation can be detected even at the level of individual electrodes. Second, compared with baseline, theta power tends to decrease and gamma power tends to increase during the presentation of all items, but these changes are amplified during the presentation of successfully encoded items. 
Changes in phase synchrony during encoding

To assess whether the observed memoryrelated power fluctuations represent phase-synchronous oscillations, we spatially localized changes in phase synchrony during memory formation to specific anatomical areas using both an ROI approach and a voxel-based graphtheoretic metric. Both approaches reduce the complex feature space of pairwise electrode interactions to extract memoryspecific information and complement each other to build a more complete picture of phase-synchronous activity during memory encoding.

To assess spatiotemporal changes in phase synchrony associated with successful memory encoding, we defined ROIs based on anatomical lobes of the brain and calculated phase synchrony between each ROI pair (see Materials and Methods). We compared these memory-related changes in phase synchrony with memoryrelated changes in spectral power in each ROI (Fig. 5). During successful memory encoding, we found that theta phase synchrony demonstrated significant initial increases in the occipital and temporal lobes that subsequently rapidly spread to a distributed set of ROI pairs and eventually concentrated in the left frontal lobe. This initial increase in theta synchrony was immediately followed by a decrease in both theta synchrony and power that spatially progressed in a similar posterior-to-anterior manner. Significant changes in gamma synchrony during encoding were limited to small areas of decreased gamma phase synchrony, which was surprising given the widespread increase in gamma power that occurred simultaneously during memory formation.

Using such ROIs to spatially aggregate phase-synchronous interactions across patients clearly demonstrates that memory encoding modulates oscillatory phase synchrony, particularly in the theta frequency band. However, a drawback of this approach is that it fails to leverage the principal advantage of ECoG over other modalities, such as scalp EEG or magnetoencephalography (MEG): very high spatial resolution. This is particularly important when investigating gamma phase synchrony, which is correlated on a much finer spatial scale than theta activity (Logothetis et al., 2007).

To circumvent this problem, we aggregated pairwise network connections using precisely defined spherical voxels and a graph-theoretic metric. As illustrated for a single participant (TJUH 11; Fig. 6), we first compared the distribution of phase-locking values observed during successful versus Successful memory encoding. area $21 ; 500-1500 \mathrm{~ms}$.

\section{A Theta SME $(3-8 \mathrm{~Hz})$}
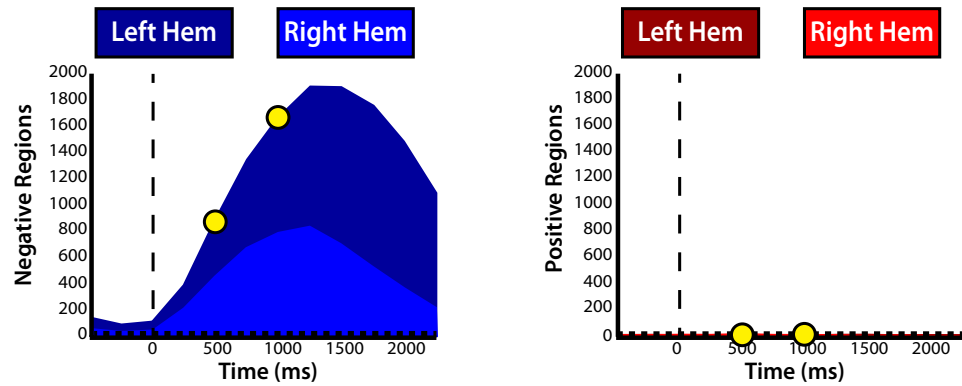

B Gamma SME (45-95 Hz)
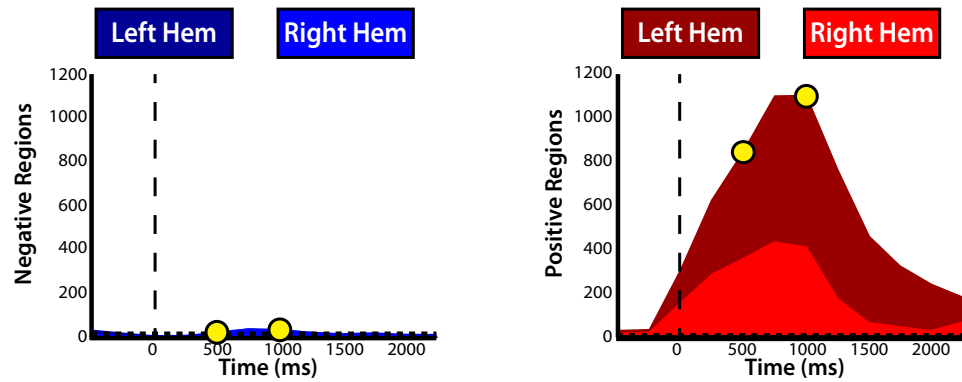

Figure 3. Temporal evolution of changes in theta and gamma power. The total number of regions exhibiting a significant decrease (left) or increase (right) in both theta $(\boldsymbol{A})$ and gamma $(\boldsymbol{B})$ power are displayed across time. Yellow circles represent the early and late word presentation intervals shown in Figure 2. Chance level $(p=0.001)$ is represented by the horizontal dashed line. The percentage of the regions in each hemisphere is proportional to the area of the light and dark colors, as indicated. SME,
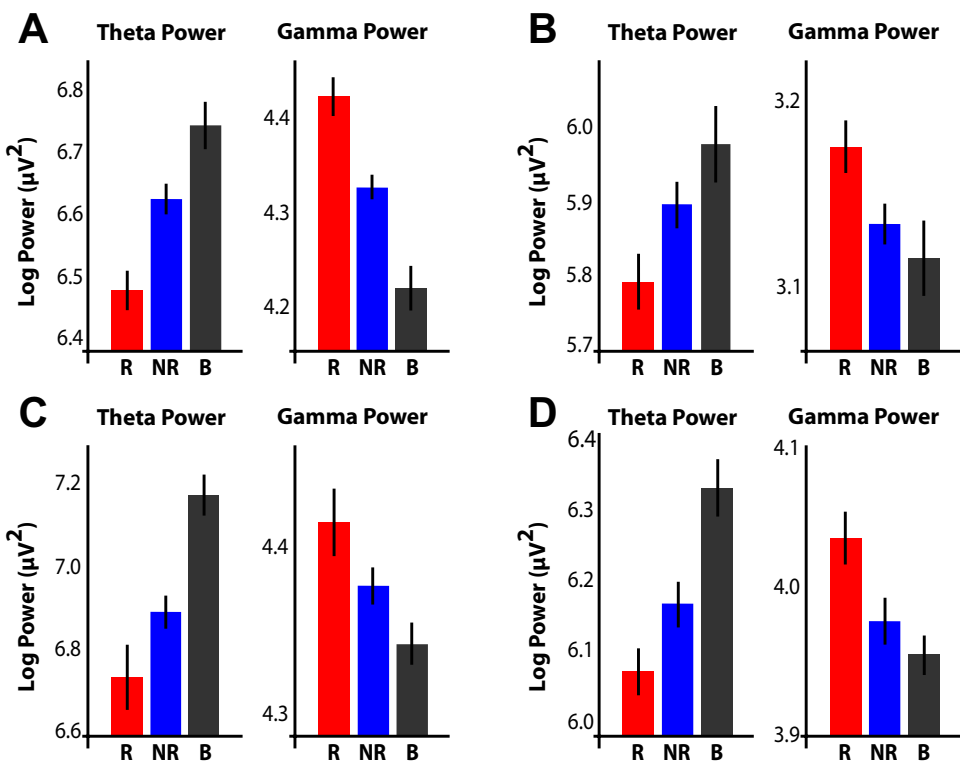

Figure 4. Examples of theta and gamma power fluctuations during successful memory formation. For four different electrodes, raw log-transformed power in the theta $(3-8 \mathrm{~Hz})$ and gamma $(45-95 \mathrm{~Hz})$ bands is shown for all subsequently recalled ( $\mathrm{R}$; red), subsequently not recalled (NR; blue), and baseline ( $B$; gray) events. Error bars represent the $95 \%$ confidence interval. $\boldsymbol{A}$, Patient TJUH 11; left Brodmann area 20; $500-1500$ ms. B, Patient TJUH 19; right posterior parahippocampal gyrus (depth electrode); $250-1250$ ms. C, Patient TJUH 23; right Brodmann area 20; 500-1500 ms. D, Patient TJUH 24; right Brodmann

unsuccessful memory encoding between each electrode pair. We used significant increases and decreases in phase synchrony to create a spatial synchrony map of task-related changes in phase synchrony for every temporal epoch for every participant. To 


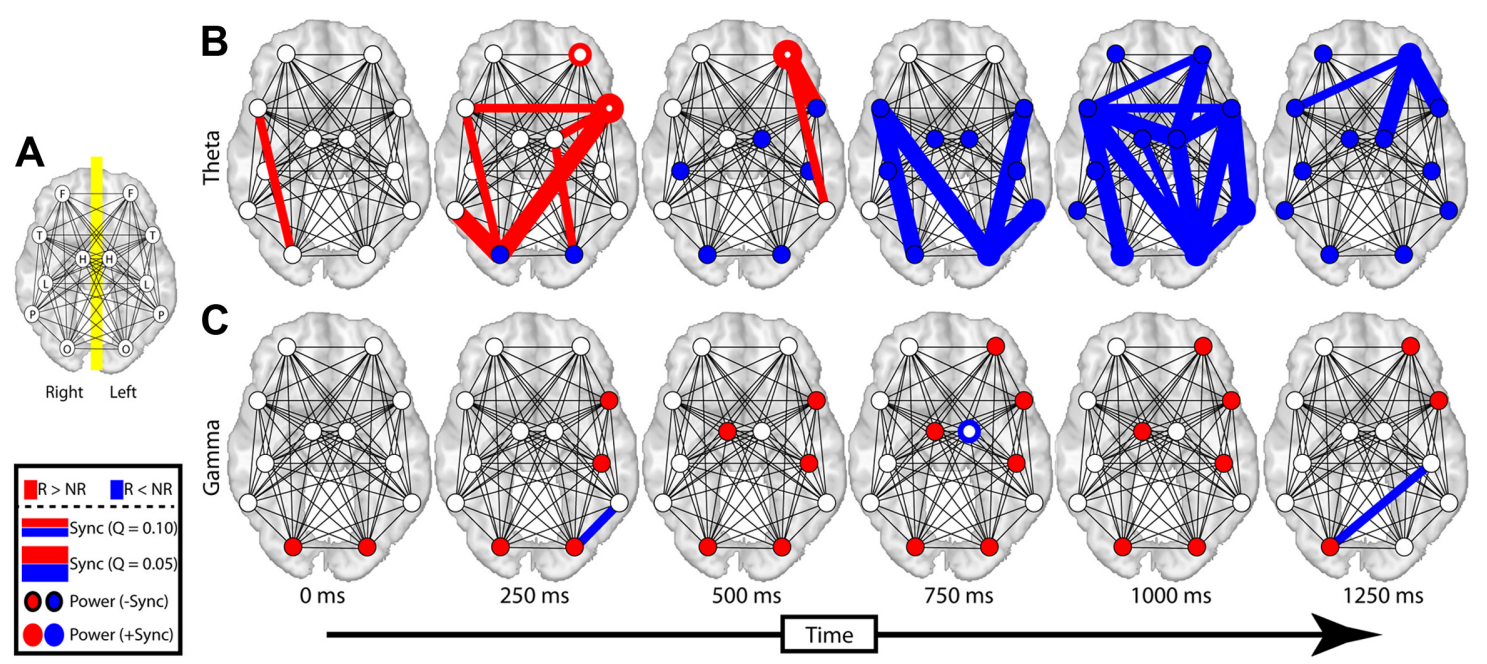

Figure 5. Change in theta and gamma lobe-wise synchrony during memory encoding. $A$, Inter- and intra-lobe synchrony was calculated across all patients for frontal (F), temporal (T), occipital $(0)$, limbic nonhippocampal (L), hippocampal $(H)$, and parietal $(P)$ lobes. The resulting lobe-wise synchrony is displayed separately for theta $(\boldsymbol{B})$ and gamma $(\boldsymbol{C})$ frequency bands. Increases and decreases in synchrony are shown in red and blue, respectively. N, Not recalled; $R$, recalled. Thick and thin lines correspond to a conservative $(Q=0.05)$ and liberal $(Q=0.10)$ FDR correction for multiple comparisons, respectively. Analogous changes in power for each lobe are displayed by filling each circle using an identical color scheme. ROls exhibiting a significant change in intra-lobe synchrony are displayed by coloring the outline of each circle. Power changes could occur either in the presence ( + Sync) or absence ( - Sync) of such intra-lobe synchrony. Times indicate the midpoint of each 1000 ms time epoch represented in each column.

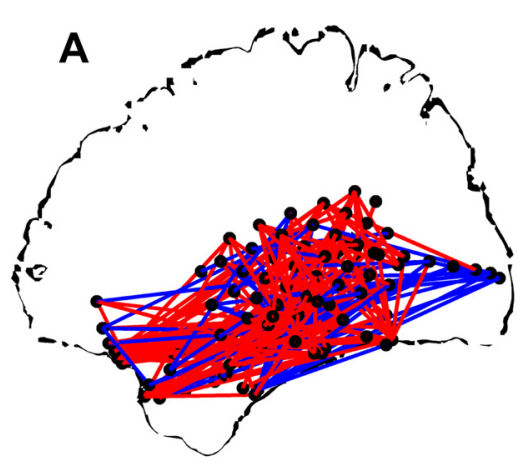

$0-1000 \mathrm{~ms}$

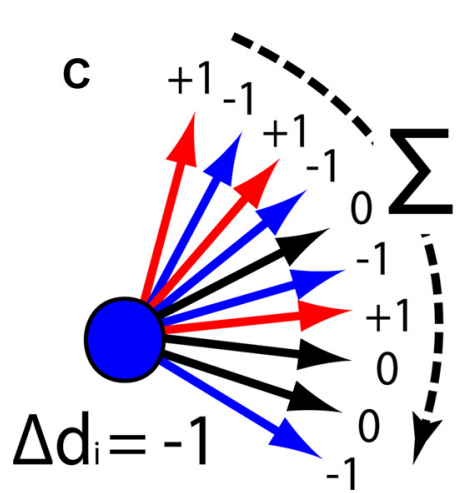

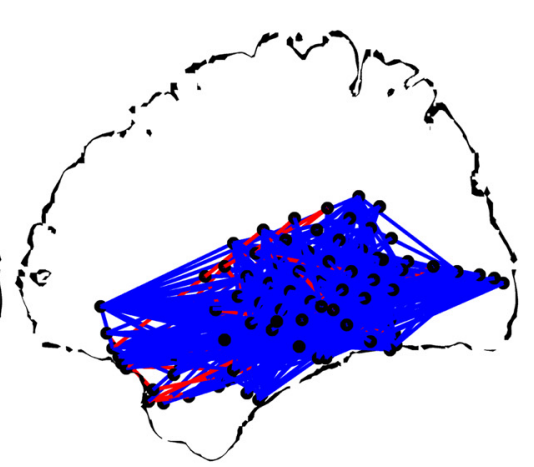

$750-1750 \mathrm{~ms}$

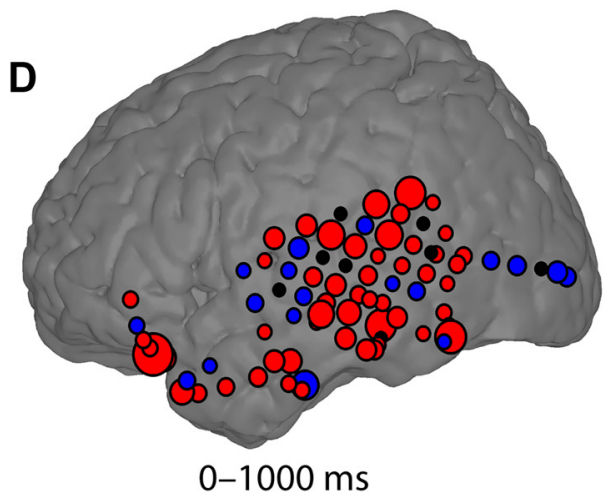

B
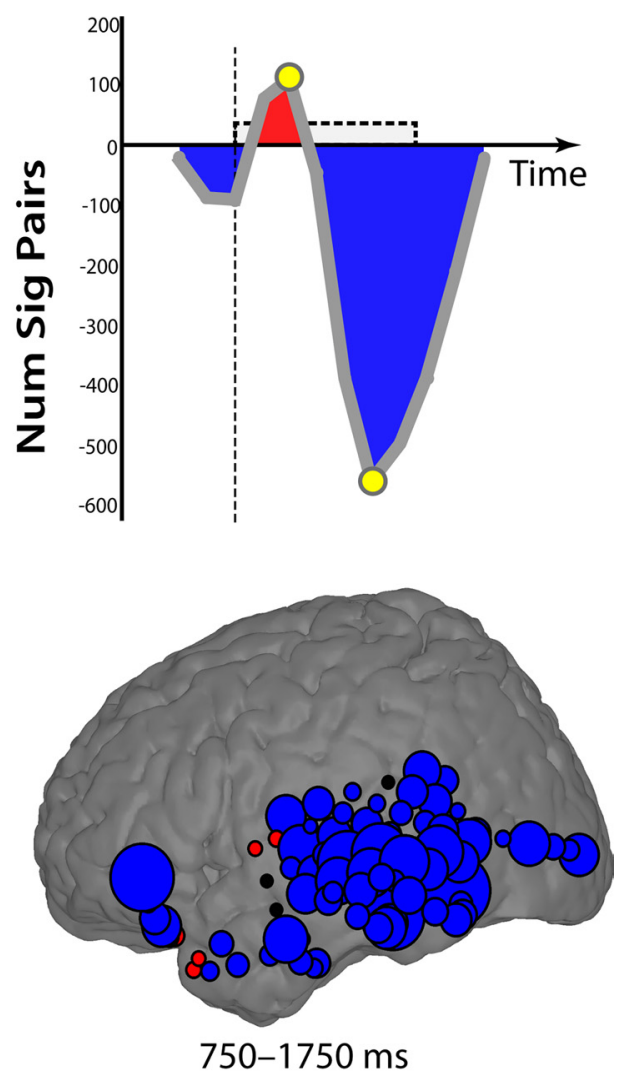

Figure 6. Aggregating pairwise network connections. A, All pairs of electrodes with significant $(p<0.05)$ increases (red lines) or decreases (blue lines) in theta synchrony during successful encoding are shown for two different time epochs for patient TJUH 11. B, For all time epochs, the difference between the total number of pairs exhibiting a significant increase and decrease in synchrony is shown. Yellow circles mark the epochs depicted in $A$. C, The change in degree, $\Delta d_{p}$ is found by tabulating significant increases or decreases in synchrony for each connection of each electrode. $D$, The change in degree, $\Delta d_{p}$, for each electrode is shown, summarizing the changes in synchrony observed in $A$. Electrodes that exhibited an overall increase or decrease in synchrony with all other electrodes during memory formation are colored in red or blue, respectively. The size of each electrode is proportional to the number of connections demonstrating significant changes in synchrony.

examine the temporal evolution of these changes, we collapsed the spatial information contained in each synchrony map into a single value by subtracting the number of electrode pairs exhibiting a statistically significant $(p<0.05)$ decrease in phase syn- chrony during successful encoding from the number of pairs exhibiting a significant increase (Fig. 6B). For this participant, there was a brief increase in theta synchrony immediately after word presentation, followed by a much larger decrease in theta 

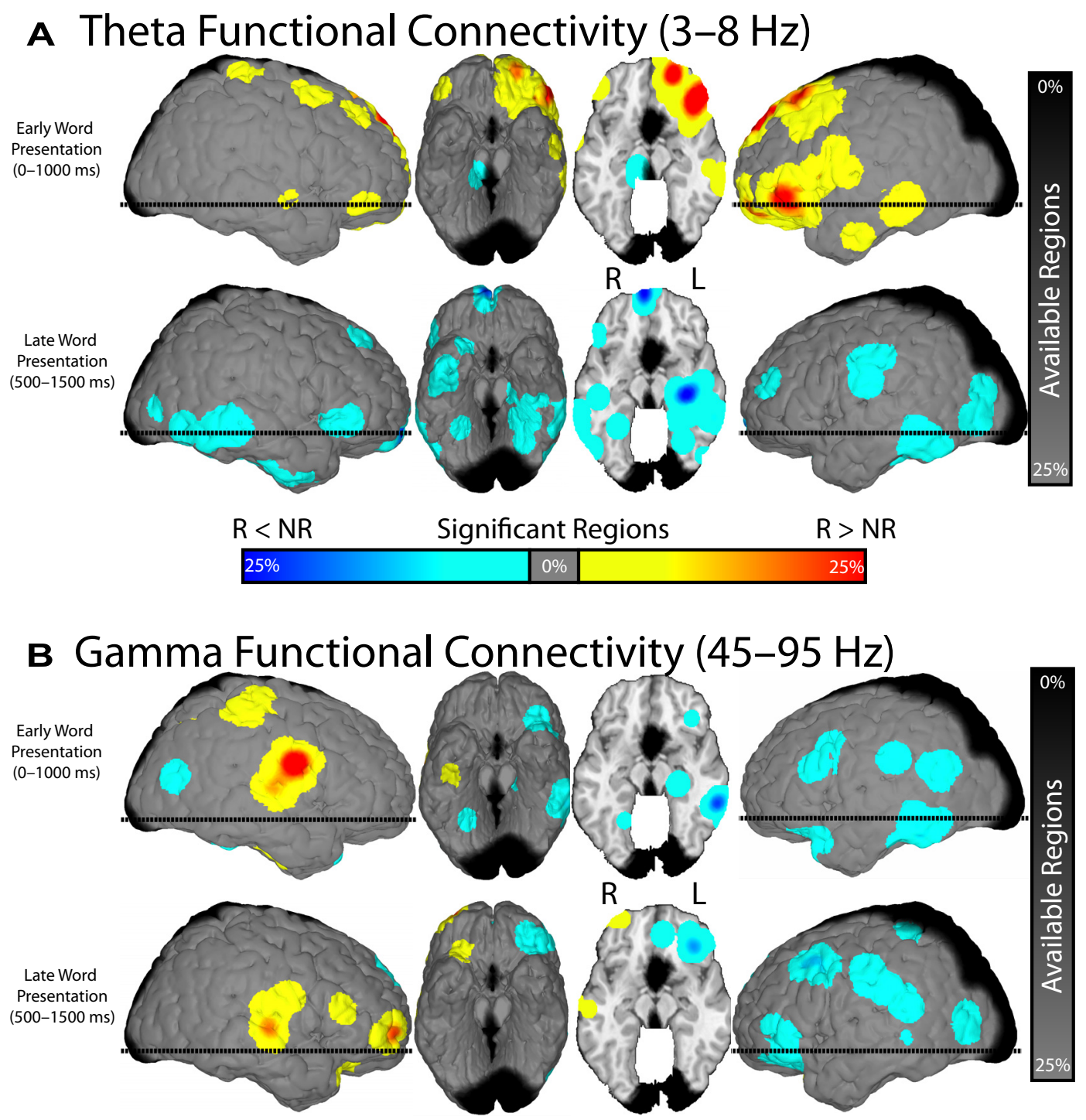

Figure 7. Change in theta and gamma degree across anatomical locations. All spherical regions that exhibited a significant $(p<0.001)$ change in theta $(\boldsymbol{A})$ and gamma $(\boldsymbol{B})$ degree $\left(\Delta d_{p}\right)$ during successful memory encoding are displayed on a standardized three-dimensional brain. N, Not recalled; R, recalled. Increases and decreases in phase synchrony are shown in red and blue, respectively. The color scale and grayscale reflect the percentage of surrounding regions with identical encoding-related effects and with more than five patients, as in Figure 2. L, Left; R, right.

phase synchrony at the end of word presentation, mirroring the effects seen among ROIs across participants.

To more precisely spatially localize changes in phase synchrony during memory encoding, we used a graph-theoretic approach. Briefly, we designated every electrode, $e_{p}$, as a node in a larger network and calculated the total number of other nodes in the network, $e_{q}$, that share a statistically significant increase in synchrony with that node minus the total number of other nodes that share a statistically significant decrease in synchrony during successful memory encoding (Fig. 6C; see Materials and Methods). The resulting change in degree, $\Delta d_{p}$, represents the extent to which each node in the network increases or decreases its phase synchrony with the rest of the network during memory encoding. Defining the functional connectivity of each electrode in this manner allows us to localize anatomical areas in which memoryrelated changes in synchrony were most concentrated. Spatially localizing the changes in theta synchrony observed in Figure $6 \mathrm{~A}$ demonstrated that the temporal lobe was marked by increases in theta synchrony during early word presentation, followed by prominent decreases in theta synchrony during late word presen- tation localized to the left lateral and inferior temporal cortex (Fig. 6D).

Using this approach, we calculated the change in degree for each electrode for each participant and determined whether the changes observed in a particular region were statistically significant across all participants. As for our power analysis, we used 5484 identical spherical voxels uniformly placed across Talairach space to group nearby electrodes for each participant (Fig. 1D). We used a permutation procedure to assess whether the changes in degree for each region were statistically significant across participants during early $(0-1000 \mathrm{~ms})$ and late $(500-1500 \mathrm{~ms})$ word presentation (see Materials and Methods).

We found that successful memory encoding was marked by a reliable initial increase in theta synchrony localized to the left temporal, prefrontal, and orbitofrontal cortex (Fig. 7A). The increases in theta synchrony were not associated with a prominent increase in theta power (Fig. 2A), which suggested that power analyses alone were insufficient to isolate these phasesynchronous theta oscillations. After this initial increase, we found that theta synchrony during encoding exhibited reliable 
A Theta Functional Connectivity $(3-8 \mathrm{~Hz})$
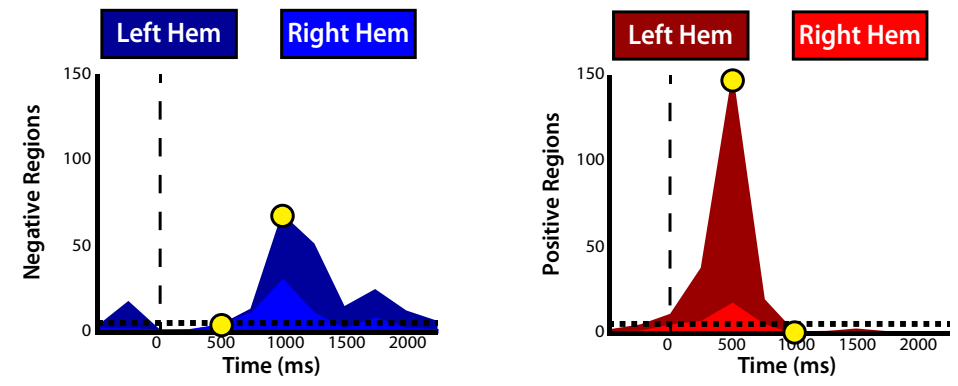

B Gamma Functional Connectivity $(45-95 \mathrm{~Hz})$
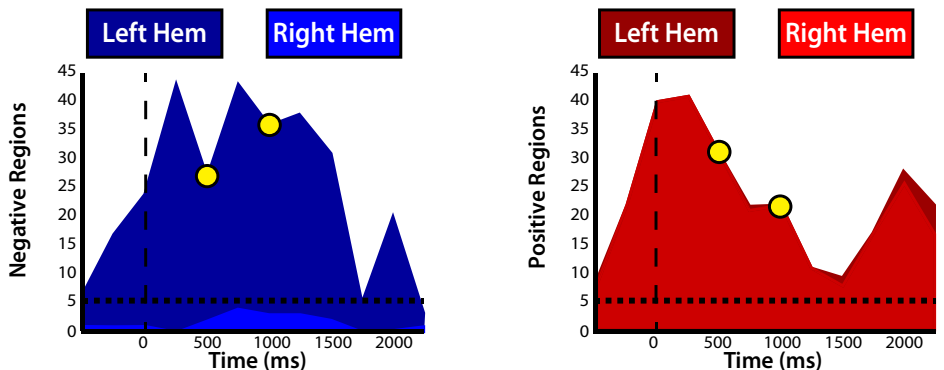

Figure 8. Temporal evolution of changes in theta and gamma degree. The total number of regions exhibiting a significant decrease (left) or increase (right) in both theta $(\boldsymbol{A})$ and gamma $(\boldsymbol{B})$ connectivity $\left(\Delta d_{p}\right)$ are displayed across time. Yellow circles represent the early and late word presentation intervals shown in Figure 7 . Chance level $(p=0.001)$ is represented by the horizontal dashed line. Hemispheric bias is represented as in Figure 3.

A

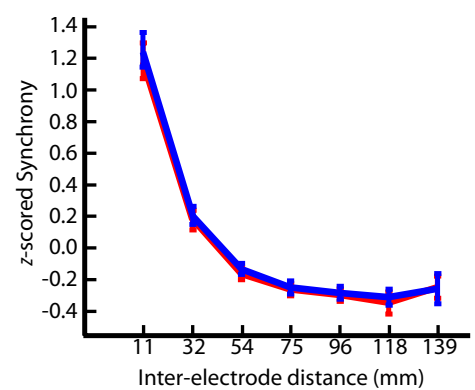

B

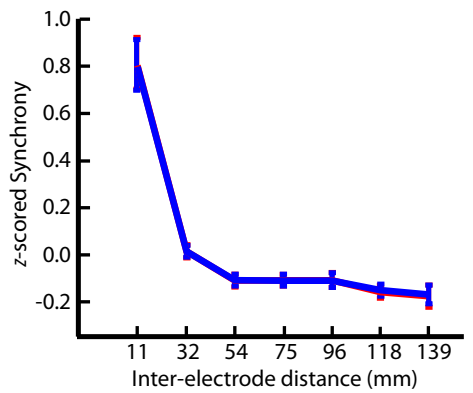

Figure 9. Normalized phase synchrony as a function of inter-electrode distance. Phase-locking values from all electrode pairs were separately $z$-scored for theta $(A)$ and gamma $(B)$ frequency bin for each patient. $z$-scored phase-locking values from recalled (red lines) and non-recalled (blue lines) items were then categorized according to inter-electrode distance using seven bins ranging from 0 to $150 \mathrm{~mm}$. The midpoint of each bin is represented on the $x$-axis. Phase-locking values within each bin were then averaged across all patients. Error bars represent $95 \%$ confidence interval across patients. A Wilcoxon's test was used to compare the recalled and non-recalled averaged phase-locking values within each bin across patients; no bins exhibited a significant ( $p<$ 0.1 ) change in averaged phase-locking values.

decreases localized diffusely throughout the brain but which were most concentrated in the left medial temporal lobe. The decrease in theta synchrony overlapped in time and space with regions in which theta power also decreased. These results extend the ROI approach to demonstrate that the left prefrontal cortex, more than any other region, is the major hub of the theta synchronous verbal episodic memory encoding network.

Conversely, when we examined changes in gamma synchrony, we found distinct regions of gamma synchrony during memory encoding (Fig. $7 B$ ). This result highlights the utility of investigating spatially precise synchronous interactions. In particular, we found that successful memory encoding involved either no change or an overall decrease in gamma synchrony in the left hemisphere. This result was surprising given the highly reliable increases in gamma power that occurred simultaneously in the same region (Fig. 2B). In addition, we found increased gamma synchrony that localized to the right hemisphere in the frontotemporal (perisylvian) areas of the brain.

Analogously to the power analysis in Figure 3, we examined the temporal evolution of changes in phase synchrony in Figure 8. For each time epoch, we separately counted the number of spherical voxels that exhibited statistically significant decreases or increases in synchrony, as measured by the change in degree, during successful memory encoding. The observed increases in theta synchrony peaked during early word presentation, whereas the decreases peaked during late word presentation. In both cases, the regions exhibiting these changes were more prominent in the left hemisphere (Fig. $8 A$ ). Additionally, we found a consistent hemispheric lateralization of gamma phase synchrony such that increases in phase synchrony were prominent in the right hemisphere and decreases in phase synchrony were prominent in the left hemisphere (Fig. $8 B)$. Although gamma synchrony was reliably modulated by memory formation, the temporal envelope defining these changes was not as well defined.

To further investigate the observed changes in phase synchrony, we examined phase synchrony between every electrode pair across all 68 patients in our database as a function of inter-electrode distance. In our graph-theoretic approach, phase synchrony between electrode pairs equally contributes to functional connectivity regardless of the distance between them. When we examined the relation with distance, however, we found that both theta and gamma phase synchrony were modulated by distance in a highly reliable manner and that this relation was independent of successful and unsuccessful encoding (Fig. 9). However, despite this relation with distance, our graphtheoretic analysis only considers relative changes in the phase-locking value between conditions. Hence, even when separated by variable distances, electrode pairs were evaluated on equal footing, which is a necessary control given this relation between neural synchrony and cortical distance (Logothetis et al., 2007).

Nevertheless, the relation between phase synchrony and interelectrode distance observed here is notable and may be useful in defining the baseline spread of theta and gamma oscillations in the human brain.

\section{Discussion}

By investigating spectral power and aggregate pairwise phase synchrony during successful memory encoding across the entire brain, our data address whether the observed changes in theta and gamma spectral power reflect oscillatory or asynchronous 

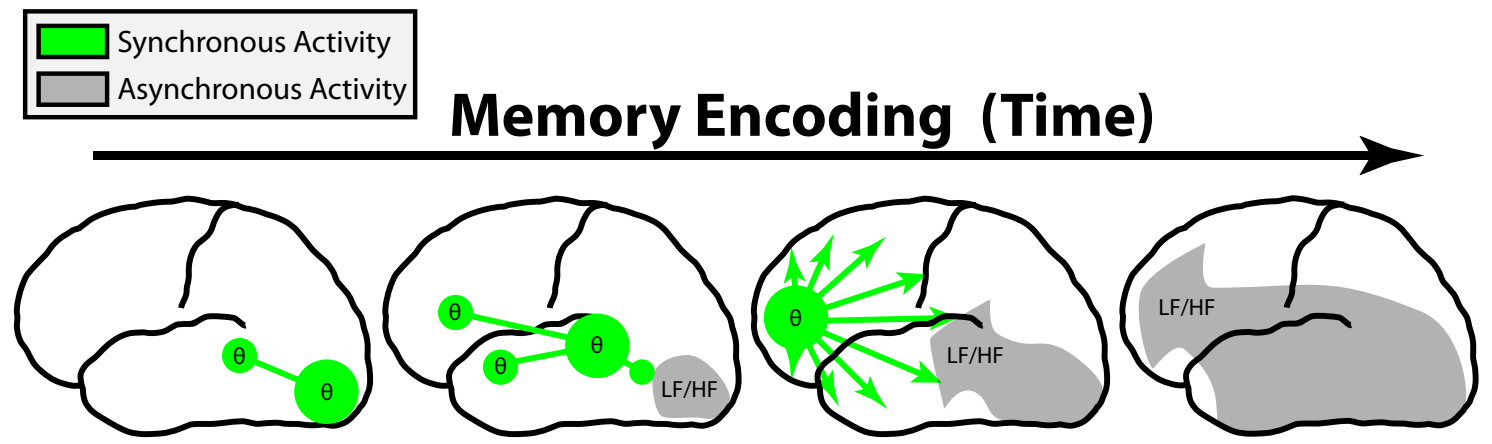

Figure 10. Summary of the ECOG verbal episodic subsequent-memory effect. In the left hemisphere immediately after word presentation, successful encoding is marked by an initial increase in theta synchrony in posterior ROls, which becomes concentrated in the left prefrontal cortex. Asynchronous activity, in both low (LF) and high (HF) frequencies, follows this increase in synchrony along a posterior-to-anterior pathway. In the right hemisphere (data not shown), the changes are more complicated and involve synchronous gamma oscillations in the right perisylvian region.

activity. In the setting of a diffuse decrease in theta power during successful memory encoding, we found an increase in theta phase synchrony early after item presentation concentrated in the left prefrontal cortex that was subsequently followed by a decrease in theta phase synchrony in the left frontal lobe and medial and lateral temporal lobes. Conversely, in the setting of a diffuse increase in gamma power, we found decreases in gamma synchrony throughout the left hemisphere and only small areas of increased synchronous gamma oscillations in the right hemisphere. The dissociation between power and phase has two major implications for the interpretation of spectral activity during memory formation. First, our data suggest that gamma activations are often asynchronous, indicating that the majority of gamma power increases during encoding do not represent temporally binding synchronous oscillations. Second, the finding that encoding involves both theta-synchrony increases and decreases occurring very near to each other in time and space helps reconcile conflicting results regarding theta power during memory formation. More generally, by building a more complete picture of the electrophysiological profile accompanying episodic memory encoding, our results help clarify the role of rhythmic neural activity in the memory system.

\section{Theta phase synchrony}

During successful memory encoding, we found increases in theta synchrony during early word presentation, supporting the hypothesis that cortical synchronized theta oscillatory activity mediates memory formation (Düzel et al., 2010; Fell and Axmacher, 2011). ROI analyses and graph-theoretic metrics localized these increases to posterior cortices, followed rapidly by increases in the left prefrontal cortex (Fig. 10). The spatially unique locus of increased theta synchrony in left prefrontal cortex suggests that this structure is highly synchronous to other regions during encoding and likely acts as the network hub of theta oscillatory activity during memory formation. This finding is consistent with previous studies demonstrating enhanced theta synchrony between prefrontal cortex and distant cortical areas during working-memory tasks (Sarnthein et al., 1998; Sauseng et al., 2004; Payne and Kounios, 2009; Liebe et al., 2012) and during episodic memory encoding and retrieval (Weiss and Rappelsberger, 2000; Fell et al., 2003; Summerfield and Mangels, 2005; Anderson et al., 2010). Although prefrontal activity may reflect contextual integration (Hyman et al., 2012), top-down interactions between cortical structures (Miller and Cohen, 2001), or communication between the neocortex and hippocampus (Jones and Wilson, 2005; Benchenane et al., 2010; Fujisawa and Buzsáki,
2011), the precise functional role of the observed synchronous theta oscillations in human episodic memory encoding remains to be determined.

After these observed increases, we found subsequent decreases in theta phase synchrony during later temporal epochs of successful memory encoding, localized to the left hemisphere and accompanied by broad anteriorly spreading decreases in theta power (Fig. 10). The degree to which memory formation involves this decrease in theta power represents perhaps the most striking finding in our data and is consistent with previous EEG and MEG findings (Sederberg et al., 2007a; Guderian et al., 2009, their Fig. 2). Such task-related decreases in low-frequency power have been traditionally classified as event-related desynchronizations (Crone et al., 1998; Pfurtscheller and Lopes Da Silva, 1999) under the assumption that they reflect a decrease in synchronized local neural activity (Singer, 1993). Here, we show that the decrease in theta power during memory formation is also accompanied by late decreases in long-range theta synchrony. This asynchronous activity may simply reflect a passive deactivation after theta synchronization, but another possibility is that the asynchronous activity itself may play a role in memory formation. Indeed, decreases in low-frequency power and synchrony correlate with the blood oxygenation level-dependent signal (Kilner et al., 2005; Niessing et al., 2005) and have been shown to covary with transitions to active cortical states (Harris and Thiele, 2011; Poulet et al., 2012). Whether memory formation represents a similar transition to a more active cortical state is unclear, but the asynchronous activity we detect here suggests this intriguing possibility.

The decreases in theta power observed during successful memory encoding in our data and in other studies (Sederberg et al., 2007a; Fell et al., 2008; Guderian et al., 2009) seem to stand in conflict with other reports of increased theta power during encoding (Klimesch et al., 1996; Mölle et al., 2002; Sederberg et al., 2003; Hanslmayr et al., 2011; Lega et al., 2011). The tendency for theta phase synchrony to both increase and decrease during successful encoding (Figs. 5-8) helps to explain this ambiguity by suggesting that theta power reflects two major types of spectral modulations: broadband low-frequency power decreases and synchronous theta oscillations. Furthermore, each of these two competing effects may be detected to a lesser or greater degree depending on the particular experimental conditions or postprocessing steps implemented.

\section{Gamma phase synchrony}

We observed increases in gamma synchrony during successful memory encoding in the right perisylvian area. This result sug- 
gests that gamma activity in this region represents narrow-band oscillations, which is generally consistent with predictions regarding the role of gamma synchrony during memory formation (Jensen et al., 2007; Jutras and Buffalo, 2010; Fell and Axmacher, 2011). Given the hypothesized role of synchronous gamma oscillations in visual attention (Tallon-Baudry and Bertrand, 1999; Fries et al., 2001; Gregoriou et al., 2009), the presence of a gamma network hub may reflect right-hemispheric attentional mechanisms (Corbetta et al., 1993; Thiebaut de Schotten et al., 2005). The location of this gamma network may seem at odds with previous work reporting increased gamma synchrony between the rhinal cortex and hippocampus during memory formation (Fell et al., 2001). However, two important features of our analyses may explain this discrepancy. First, our ROI synchrony analysis (Fig. 5) averaged electrode interactions over spatially coarse regions, and, second, our graph-theoretic approach (Fig. 6) was designed to detect hubs of network activity. Thus, synchronous oscillations limited to a single electrode pair amid all possible pairs would be difficult to detect using either approach.

By not limiting our analyses to specific anatomical circuits, we could investigate whether the widespread increases in gamma power that occur during memory formation represent phasesynchronous oscillations. We found that the most reliable increases in gamma power during memory formation were localized to the left hemisphere and were accompanied by less synchrony than expected by chance (Fig. 10). Thus, the decreases in high-frequency synchrony we observe here likely reflect asynchronous multiunit neural activity (Manning et al., 2009; Miller et al., 2009; Ray and Maunsell, 2011) or transient "bottom-up" responses (Ossandón et al., 2012) and suggest that increases in gamma power in these regions do not represent temporally binding synchronous oscillations (Jutras and Buffalo, 2010; Fell and Axmacher, 2011). Although the origins of high-frequency activations are still unclear (Crone et al., 2011; Buzsáki and Wang, 2012; Lachaux et al., 2012), our results suggest that the degree of synchrony within the gamma band can dissociate between gamma oscillations on the one hand and broadband activations on the other (Jia et al., 2011).

\section{Conclusions and future directions}

Although our analyses focus on changes in spectral power and synchrony, it is important to account for the role that evoked potentials may play in modulating phase synchrony. Trial-bytrial variability in the evoked response has been shown to interact with spectral responses and coherence measures (Wang et al., 2008; Wang and Ding, 2011). Whereas it is possible to factor out such evoked responses, doing so requires special models and analytical techniques (Truccolo et al., 2002; Xu et al., 2009). Future studies would benefit from incorporating such models to further dissociate induced synchronous activity from asynchronous evoked sources.

The presence of both synchronous and asynchronous highfrequency activity in our data is consistent with recent electrophysiological studies in both humans (Crone et al., 2011) and nonhuman primates (Ray and Maunsell, 2011) that demonstrate the segregation of gamma activity into oscillations and asynchronous processes. Similarly, the presence of both synchronous and asynchronous low-frequency activity both supports the hypothesis that low-frequency oscillations may mediate memory formation (Axmacher et al., 2006; Düzel et al., 2010; Nyhus and Curran, 2010; Fell and Axmacher, 2011) and highlights the possible role of asynchronous activity in human memory. Our analyses show that phase synchrony can be used to disentangle oscillatory from asynchronous activity in the context of the observed changes in spectral power. We found that both synchronous and asynchronous processes shape the frequency spectrum during memory formation, suggesting that theories regarding the role of spectral activity in the memory system should incorporate both asynchronous spectral patterns, as well as synchronous oscillatory activity.

\section{References}

Addison PS (2002) The illustrated wavelet transform handbook: introductory theory and applications in science, engineering, medicine and finance. Bristol, UK: Institute of Physics Publishing.

Anderson KL, Rajagovindan R, Ghacibeh GA, Meador KJ, Ding M (2010) Theta oscillations mediate interaction between prefrontal cortex and medial temporal lobe in human memory. Cereb Cortex 20:1604-1612. CrossRef Medline

Axmacher N, Mormann F, Fernández G, Elger CE, Fell J (2006) Memory formation by neuronal synchronization. Brain Res Rev 52:170-182. CrossRef Medline

Benchenane K, Peyrache A, Khamassi M, Tierney PL, Gioanni Y, Battaglia FP, Wiener SI (2010) Coherent theta oscillations and reorganization of spike timing in the hippocampal-prefrontal network upon learning. Neuron 66:921-936. CrossRef Medline

Bullmore E, Sporns O (2009) Complex brain networks: graph theoretical analysis of structural and functional systems. Nat Rev Neurosci 10:186198. CrossRef Medline

Buzsáki G, Wang XJ (2012) Mechanisms of gamma oscillations. Annu Rev Neurosci 35:203-225. CrossRef Medline

Corbetta M, Miezin FM, Shulman GL, Petersen SE (1993) A PET study of visuospatial attention. J Neurosci 13:1202-1226. Medline

Crone NE, Miglioretti DL, Gordon B, Sieracki JM, Wilson MT, Uematsu S, Lesser RP (1998) Functional mapping of human sensorimotor cortex with electrocorticographic spectral analysis. I. Alpha and beta eventrelated desynchronization. Brain 121:2271-2299. Medline

Crone NE, Korzeniewska A, Franaszczuk PJ (2011) Cortical gamma responses: searching high and low. Int J Psychophysiol 79:9-15. CrossRef Medline

Düzel E, Penny WD, Burgess N (2010) Brain oscillations and memory. Curr Opin Neurobiol 20:143-149. CrossRef Medline

Fell J, Axmacher N (2011) The role of phase synchronization in memory processes. Nat Rev Neurosci 12:105-118. CrossRef Medline

Fell J, Klaver P, Lehnertz K, Grunwald T, Schaller C, Elger CE, Fernández G (2001) Human memory formation is accompanied by rhinal-hippocampal coupling and decoupling. Nat Neurosci 4:1259-1264. CrossRef Medline

Fell J, Klaver P, Elfadil H, Schaller C, Elger CE, Fernández G (2003) Rhinalhippocampal theta coherence during declarative memory formation: interaction with gamma synchronization? Eur J Neurosci 17:1082-1088. CrossRef Medline

Fell J, Ludowig E, Rosburg T, Axmacher N, Elger CE (2008) Phase-locking within human mediotemporal lobe predicts memory formation. Neuroimage 43:410-419. CrossRef Medline

Fries P, Reynolds JH, Rorie AE, Desimone R (2001) Modulation of oscillatory neuronal synchronization by selective visual attention. Science 291: 1560-1563. CrossRef Medline

Fujisawa S, Buzsáki G (2011) A $4 \mathrm{~Hz}$ oscillation adaptively synchronizes prefrontal, VTA, and hippocampal activities. Neuron 72:153-165. CrossRef Medline

Genovese CR, Lazar NA, Nichols T (2002) Thresholding of statistical maps in functional neuroimaging using the false discovery rate. Neuroimage 15:870-878. CrossRef Medline

Gregoriou GG, Gotts SJ, Zhou H, Desimone R (2009) High-frequency, long-range coupling between prefrontal and visual cortex during attention. Science 324:1207-1210. CrossRef Medline

Guderian S, Schott BH, Richardson-Klavehn A, Düzel E (2009) Medial temporal theta state before an event predicts episodic encoding success in humans. Proc Natl Acad Sci U S A 106:5365-5370. CrossRef Medline

Hanslmayr S, Volberg G, Wimber M, Raabe M, Greenlee MW, Bäumel KH (2011) The relationship between brain oscillations and BOLD signal during memory formation: a combined EEG-fMRI study. J Neurosci 31: 15674-15680. CrossRef Medline

Harris KD, Thiele A (2011) Cortical state and attention. Nat Rev Neurosci 12:509-523. CrossRef Medline

Hyman JM, Ma L, Balaguer-Ballester E, Durstewitz D, Seamans JK (2012) 
Contextual encoding by ensembles of medial prefrontal cortex neurons. Proc Natl Acad Sci U S A 109:5086-5091. CrossRef Medline

Jensen O, Kaiser J, Lachaux JP (2007) Human gamma-frequency oscillations associated with attention and memory. Trends Neurosci 30:317324. CrossRef Medline

Jia X, Smith MA, Kohn A (2011) Stimulus selectivity and spatial coherence of gamma components of the local field potential. J Neurosci 31:9390-9403. CrossRef Medline

Jones MW, Wilson MA (2005) Phase precession of medial prefrontal cortical activity relative to the hippocampal theta rhythm. Hippocampus 15: 867-873. CrossRef Medline

Jutras MJ, Buffalo EA (2010) Synchronous neural activity and memory formation. Curr Opin Neurobiol 20:150-155. CrossRef Medline

Kahana MJ (2006) The cognitive correlates of human brain oscillations. J Neurosci 26:1669-1672. CrossRef Medline

Kahana MJ (2012) Foundations of human memory. New York: Oxford UP.

Kilner JM, Mattout J, Henson R, Friston KJ (2005) Hemodynamic correlates of EEG: a heuristic. Neuroimage 28:280-286. CrossRef Medline

Klimesch W, Doppelmayr M, Russegger H, Pachinger T (1996) Theta band power in the human scalp EEG and the encoding of new information. Neuroreport 7:1235-1240. CrossRef Medline

Lachaux JP, Rodriguez E, Martinerie J, Varela FJ (1999) Measuring phase synchrony in brain signals. Hum Brain Mapp 8:194-208. CrossRef Medline

Lachaux JP, Axmacher N, Mormann F, Halgren E, Crone NE (2012) Highfrequency neural activity and human cognition: past, present and possible future of intracranial eeg research. Prog Neurobiol 98:279-301. CrossRef Medline

Lancaster JL, Woldorff MG, Parsons LM, Liotti M, Freitas CS, Rainey L, Kochunov PV, Nickerson D, Mikiten SA, Fox PT (2000) Automated Talairach atlas labels for functional brain mapping. Hum Brain Mapp 10:120-131. CrossRef Medline

Lega BC, Jacobs J, Kahana M (2012) Human hippocampal theta oscillations and the formation of episodic memories. Hippocampus 22:748-761. CrossRef Medline

Liebe S, Hoerzer GM, Logothetis NK, Rainer G (2012) Theta coupling between V4 and prefrontal cortex predicts visual short-term memory performance. Nat Neurosci 15:456-462, S1-S2. CrossRef Medline

Logothetis NK, Kayser C, Oeltermann A (2007) In vivo measurement of cortical impedance spectrum in monkeys: implications for signal propagation. Neuron 55:809-823. CrossRef Medline

Maldjian JA, Laurienti PJ, Kraft RA, Burdette JH (2003) An automated method for neuroanatomic and cytoarchitectonic atlas-based interrogation of fMRI data sets. Neuroimage 19:1233-1239. CrossRef Medline

Manning JR, Jacobs J, Fried I, Kahana MJ (2009) Broadband shifts in LFP power spectra are correlated with single-neuron spiking in humans. J Neurosci 29:13613-13620. CrossRef Medline

Manning JR, Polyn SM, Baltuch GH, Litt B, Kahana MJ (2011) Oscillatory patterns in temporal lobe reveal context reinstatement during memory search. Proc Natl Acad Sci U S A 108:12893-12897. CrossRef Medline

Miller EK, Cohen JD (2001) An integrative theory of prefrontal cortex function. Annu Rev Neurosci 24:167-202. CrossRef Medline

Miller KJ, Zanos S, Fetz EE, den Nijs M, Ojemann JG (2009) Decoupling the cortical power spectrum reveals real-time representation of individual finger movements in humans. J Neurosci 29:3132-3137. CrossRef Medline

Mölle M, Marshall L, Fehm HL, Born J (2002) EEG theta synchronization conjoined with alpha desynchronization indicate intentional encoding. Eur J Neurosci 15:923-928. CrossRef Medline

Niessing J, Ebisch B, Schmidt KE, Niessing M, Singer W, Galuske RA (2005) Hemodynamic signals correlate tightly with synchronized gamma oscillations. Science 309:948-951. CrossRef Medline

Nunez PL, Srinivasan R (2006) Electric fields of the brain. New York: Oxford UP.

Nyhus E, Curran T (2010) Functional role of gamma and theta oscillations in episodic memory. Neurosci Biobehav Rev 34:1023-1035. CrossRef Medline

Ossandón T, Vidal JR, Ciumas C, Jerbi K, Hamam é CM, Dalal SS, Bertrand O, Minotti L, Kahane P, Lachaux JP (2012) Efficient "pop-out" visual search elicits sustained broadband gamma activity in the dorsal attention network. J Neurosci 32:3414-3421. CrossRef Medline
Paller KA, Wagner AD (2002) Observing the transformation of experience into memory. Trends Cogn Sci 6:93-102. CrossRef Medline

Payne L, Kounios J (2009) Coherent oscillatory networks supporting shortterm memory retention. Brain Res 1247:126-132. CrossRef Medline

Pfurtscheller G, Lopes da Silva FH (1999) Event-related EEG/MEG synchronization and desynchronization: basic principles. Clin Neurophysiol 110:1842-1857. CrossRef Medline

Poulet JF, Fernandez LM, Crochet S, Petersen CC (2012) Thalamic control of cortical states. Nat Neurosci 15:370-372. CrossRef Medline

Ray S, Maunsell JH (2010) Differences in gamma frequencies across visual cortex restrict their possible use in computation. Neuron 67:885-896. CrossRef Medline

Ray S, Maunsell JH (2011) Different origins of gamma rhythm and highgamma activity in macaque visual cortex. PLoS Biol 9:e1000610. CrossRef Medline

Sarnthein J, Petsche H, Rappelsberger P, Shaw GL, von Stein A (1998) Synchronization between prefrontal and posterior association cortex during human working memory. Proc Natl Acad Sci U S A 95:7092-7096. CrossRef Medline

Sauseng P, Klimesch W, Doppelmayr M, Hanslmayr S, Schabus M, Gruber WR (2004) Theta coupling in the human electroencephalogram during a working memory task. Neurosci Lett 354:123-126. CrossRef Medline

Sederberg PB, Kahana MJ, Howard MW, Donner EJ, Madsen JR (2003) Theta and gamma oscillations during encoding predict subsequent recall. J Neurosci 23:10809-10814. Medline

Sederberg PB, Schulze-Bonhage A, Madsen JR, Bromfield EB, McCarthy DC, Brandt A, Tully MS, Kahana MJ (2007a) Hippocampal and neocortical gamma oscillations predict memory formation in humans. Cereb Cortex 17:1190-1196. CrossRef Medline

Sederberg PB, Schulze-Bonhage A, Madsen JR, Bromfield EB, Litt B, Brandt A, Kahana MJ (2007b) Gamma oscillations distinguish true from false memories. Psychol Sci 18:927-932. CrossRef Medline

Serruya M, Sederberg P, Kahana M (2012) Power shifts track serial position and modulate encoding in human episodic memory. Cereb Cortex. Advance online publication. Retrieved November 25, 2012. doi: 10.1093/cercor/bhs318. CrossRef Medline

Siegel M, Donner TH, Engel AK (2012) Spectral fingerprints of large-scale neuronal interactions. Nat Rev Neurosci 13:121-134. CrossRef Medline

Singer W (1993) Synchronization of cortical activity and its putative role in information processing and learning. Annu Rev Physiol 55:349-374. CrossRef Medline

Summerfield C, Mangels JA (2005) Coherent theta-band EEG activity predicts item-context binding during encoding. Neuroimage 24:692-703. CrossRef Medline

Tallon-Baudry C, Bertrand O (1999) Oscillatory gamma activity in humans and its role in object representation. Trends Cogn Sci 3:151-162. CrossRef Medline

Thiebaut de Schotten M, Urbanski M, Duffau H, Volle E, Lévy R, Dubois B, Bartolomeo P (2005) Direct evidence for a parietal-frontal pathway subserving spatial awareness in humans. Science 309:2226-2228. CrossRef Medline

Truccolo WA, Ding M, Knuth KH, Nakamura R, Bressler SL (2002) Trialto-trial variability of cortical evoked responses: implications for the analysis of functional connectivity. Clin Neurophysiol 113:206-226. CrossRef Medline

Varela F, Lachaux JP, Rodriguez E, Martinerie J (2001) The brainweb: phase synchronization and large-scale integration. Nat Rev Neurosci 2:229-239. CrossRef Medline

Wang X, Ding M (2011) Relation between p300 and event-related thetaband synchronization: a single-trial analysis. Clin Neurophysiol 122: 916-924. CrossRef Medline

Wang X, Chen Y, Ding M (2008) Estimating granger causality after stimulus onset: a cautionary note. Neuroimage 41:767-776. CrossRef Medline

Weiss S, Rappelsberger P (2000) Long-range EEG synchronization during word encoding correlates with successful memory performance. Cogn Brain Res 9:299-312. CrossRef Medline

Xu L, Stoica P, Li J, Bressler SL, Shao X, Ding M (2009) ASEO: a method for the simultaneous estimation of single-trial event-related potentials and ongoing brain activities. IEEE Trans Biomed Eng 56:111-121. CrossRef Medline 